\title{
Deutschland: Gutachten des WBP zur Gestaltherapie
}

\author{
Peter Schulthess
}

In der letzten Ausgabe von à jour!, «Psychotherapie-Berufsentwicklung», wurde über das Gutachten des deutschen Wissenschaftlichen Beirates Psychotherapie (WBP) vom Dezember 2017 zur humanistischen Psychotherapie berichtet. Die Gestalttherapie war darin als ein psychotherapeutischer Ansatz unter den Verfahren der $\mathrm{Hu}$ manistischen Psychotherapie mitbeurteilt worden. Die Frage, ob Gestalttherapie im Sinne des deutschen Psychotherapie Gesetzes (PsychThG) als ein eigenes Verfahren gelten könnte, wurde dort nicht geklärt, weil der entsprechende Antrag gar nicht gestellt worden war. Die beteiligten humanistischen Psychotherapierichtungen versuchten, die «Humanistische Psychotherapie» als Verfahren anerkennen zu lassen, was der WBP nicht tat. Die Begründung lautete, «Humanistische Psychotherapie» sei kein einheitliches Verfahren, viel mehr ein Sammelbegriff für verschiedene Therapierichtungen in der Tradition der humanistischen Psychologie.

Im Juni 2018 hatte der WBP nun in einem weiteren Gutachten zu entscheiden, ob Gestalttherapie als Verfahren wissenschaftlich anerkannt werden kann. Ein entsprechendes Gesuch war vom Deutschen Dachverband Gestalttherapie für approbierte Psychotherapeuten (DDGAP) unter der Federführung von Lotte Hartmann-Kottek eingereicht worden. Da Gestalttherapie auch im Antrag «Humanistische Psychotherapie» enthalten war, hatte man die Beurteilung dieses Antrages vorerst bis zum Abschluss ersteren Verfahrens ausgesetzt.

Der WBP kam in einem ersten Schritt zum Schluss, dass es sich bei der Gestalttherapie um ein Verfahren entsprechend den Kriterien des Methodenpapiers des WBP handle.

In einem zweiten Schritt wurde die Wirksamkeit der Gestalttherapie anhand der eingereichten Studien geprüft. Diese Prüfung erfolgt getrennt nach Störungsbereich, da der WBP davon ausgeht, dass die Wirksamkeit in einem Störungsbereich nicht den Schluss zulässt, dass ein Verfahren in anderen Anwendungsbereichen ebenso wirksam ist. Im Bereich "affektive Störungen» gilt die Gestalttherapie als wissenschaftlich anerkanntes Verfahren, da (lediglich) drei Studien aus der Forschergruppe um Lesley Greenberg zur Emotionsfokussierten Psychotherapie (EFT) anerkannt wurden. Forschung zur EFT wird vom WBP sowohl für EFT, als auch für Gesprächspsychotherapie und Gestalttherapie angerechnet, da es bei diesen grosse Gemeinsamkeiten mit der EFT gibt. Im Anwendungsbereich «Angst- und Zwangsstörungen» wurde die einzige berücksichtigte Studie abgelehnt, da sie keine Überlegenheit der EFT gegenüber der Placebo-Kontrollgruppe zeigen konnte.

Auch eine methodisch ausreichende Studie im Anwendungsbereich «somatoforme und dissoziative Störungen» wurde abgelehnt, da keine Überlegenheit gegenüber den Kontrollbedingungen gezeigt werden konnte. Im Bereich «Anpassungsund Belastungsstörungen» wurde eine Studie von Butollo als Wirksamkeitsnachweis für die Gestalttherapie anerkannt. Im Bereich «psychische und soziale Faktoren bei somatischen Erkrankungen» wurden zwei berücksichtigte Studien zur EFT als Wirksamkeitsnachweise anerkannt, zwei weitere methodisch ausreichende Studien aber abgelehnt. Im Bereich «gemischte Störungen» wurde eine Studie von Paivio und Nieuwenhuis als Wirksamkeitsnachweis für EFT anerkannt. Abschliessend fasst der WBP seine Einschätzung folgendermassen zusammen: «Bei Erwachsenen kann nach den vom WBP entwickelten Kriterien für Gestalttherapie im Anwendungsbereich «affektive Störungen〉 die wissenschaftliche Anerkennung festgestellt werden.»

Bei Kindern und Jugendlichen kann diese hingegen nicht erfolgen, da keine entsprechenden Studien vorgelegt wurden und auch in einer umfangreichen Literaturrecherche nicht identifiziert werden konnten.

In einem dritten Schritt prüfte der WBP, ob die Gestalttherapie als vertiefte Ausbildung zugelassen werden kann und verneint diese Frage, weil lediglich im Bereich «affektive Störungen» bei Erwachsenen die wissenschaftliche Anerkennung festgestellt werden konnte. Um als Verfahren für die vertiefte Ausbildung anerkannt $\mathrm{zu}$ werden, müssten für mindestens drei Anwendungsbereiche (oder gar vier, je nach Bereichen) jeweils mindestens drei Studien anerkannt sein. Erneut ist darauf hinzuweisen, dass die Kriterien des WBP, ob Studien überhaupt berücksichtigt werden, höchst problematisch sind. Von 313 eingereichten Studien wurden für die Gestalttherapie lediglich 21 Studien voll bewertet. Der Rest fiel nach Prüfung der vom WBP selber festgelegten methodischen Mindestkriterien ausser Betracht.

Peter Schulthess ist Vorstandsmitglied der ASP.

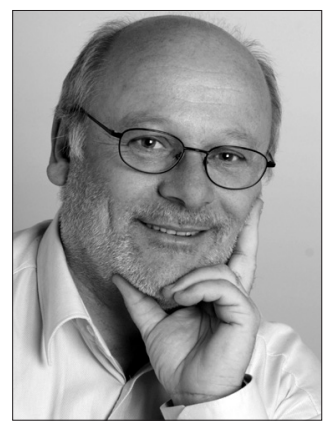

